\title{
Dialysis and end of life: an analysis of advance care planning and patient outcomes
}

\author{
Authors: Heena Khiroya, ${ }^{A *}$ Sonia Miller ${ }^{A}$ and Jyoti Baharani ${ }^{A}$
}

\section{Introduction}

The End of Life Care in Advanced Kidney Disease Framework suggests that renal units should create a renal supportive care register (RSCR) to promote consistent communication with patients and to encourage advance care planning. ${ }^{1}$ The aim of the RSCR at Birmingham Heartlands Hospital is to identify patients who are requiring dialysis with a prognosis of less than 12 months. This work aims to explore whether patients were identified appropriately on the RSCR, and if conversations around withdrawal of dialysis and end of life took place.

\section{Methods}

We reviewed the inpatient and outpatient consultations of patients who died while listed on the RSCR between 1 January 2016 and 31 December 2018. We recorded the dates when patients were added to the RSCR and when they died. We reviewed conversations around dialysis withdrawal and events at the end of life.

\section{Results and discussion}

Data from Proton, the renal team's coding system, showed that there were 80 deaths of patients listed on the RSCR: 59\% were male, $41 \%$ were female. The median age at death was 77.5 years (interquartile range (IQR) 12.25 years). Thirty-eight per cent of these patients had an alert on Concerto, the hospital's main electronic system, informing users that the patient was on the RSCR.

Eighty-eight per cent of patients were listed on the RSCR within 12 months of death; $69 \%$ of these were listed on the day they died. For the remaining patients who were listed on the register, Fig 1 illustrates that the median time to death from being placed on the register was 1.75 months (IQR 7.54 months).

Thirty-eight per cent of patients were offered a conversation on withdrawal of dialysis; $70 \%$ of these then opted to withdraw. Cited reasons for continuing dialysis after these conversations were families' refusal to accept palliation and denial. Of those who did not have dialysis formally withdrawn prior to death, there were reports of dialysis being withheld due to low blood pressure and patients being too unwell to come in from home for dialysis.

Eighty-seven per cent had valid 'do not attempt cardiopulmonary resuscitation' (DNACPR) forms. Two patients

Authors: ${ }^{A}$ University Hospitals Birmingham NHS Foundation Trust, Birmingham, UK; *RCP chief registrar

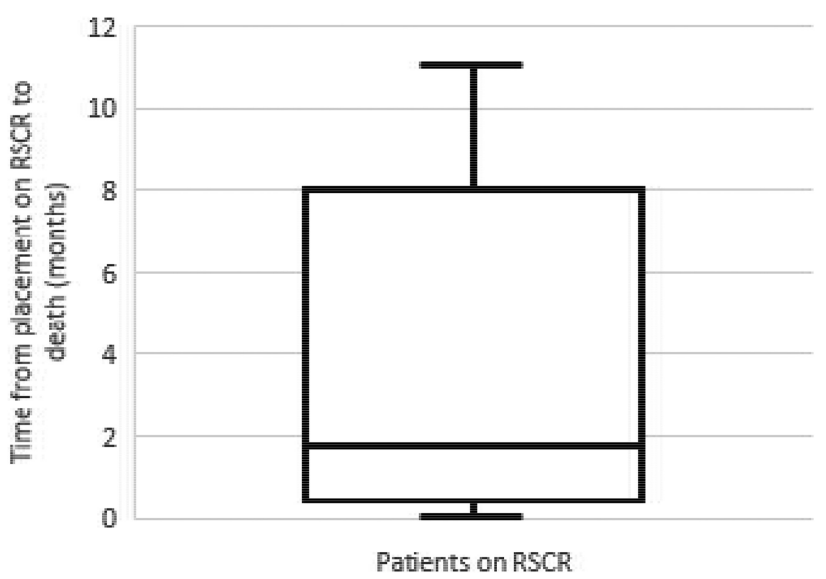

Fig 1. Time to death in months from being placed on the renal supportive care register (RSCR).

who did not have DNACPR forms received CPR (without return of spontaneous circulation) on the day of their death in hospital. Preferred place of death (PPD) was established in $20 \%$ of patients (Fig 2). While the majority of patients asked chose their PPD as home, $65 \%$ of patients on the RSCR died in hospital.

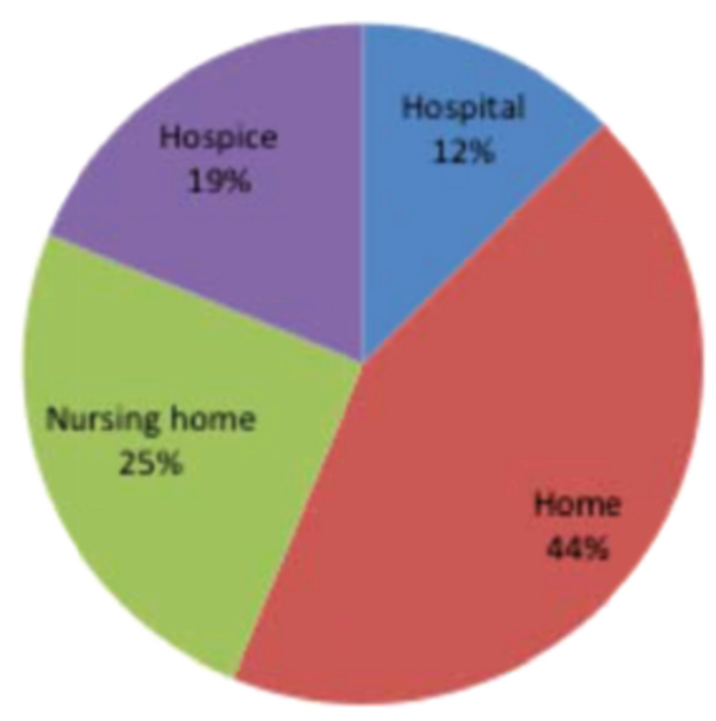

Fig 2. Preferred place of death. 
We recommend that all patients on the RSCR should have alerts placed on Concerto. This would ensure that the wider hospital, who may not know the patient as well as the renal team, are prompted to think about advance care planning. The literature reinforces that alerts can improve healthcare professionals' engagement with conversations around resuscitation. ${ }^{2}$

\section{Conclusion}

Our data suggests that the deterioration of these patients may have been unrecognised. While some deaths are likely to be unexpected, we are missing opportunities to engage patients with end-stage renal disease in advance care planning.

\section{Conflicts of interest}

None declared.

\section{References}

1 NHS. End of life care in advanced kidney disease: a framework for implementation. NHS: 2015. www.england.nhs.uk/improvementhub/wp-content/uploads/sites/44/2017/11/Advanced-kidneydisease.pdf [Accessed 16 July 2019].

2 Garcia CA, Bhatnagar M, Rodenbach R et al. Standardization of Inpatient CPR Status Discussions and Documentation Within the Division of Hematology-Oncology at UPMC Shadyside: Results From PDSA Cycles 1 and 2. J Oncol Pract 2019;15:e746-54. 